\title{
Population genetics of Trypanosoma brucei circulating in Glossina palpalis palpalis and domestic animals of the Fontem sleeping sickness focus of Cameroon
}

\author{
Gustave Simo ${ }^{1 *}$, Guy Roger Njitchouang ${ }^{2}$, Tresor Tito Tanekou Melachio ${ }^{2}$, Flobert Njiokou ${ }^{2}$, Gerard Cuny ${ }^{3}$ \\ and Asonganyi Tazoacha ${ }^{4}$
}

\begin{abstract}
Background: Human African Trypanosomiasis is still a public health threat in Cameroon. To assess Trypanosoma brucei strains circulating in the Fontem sleeping sickness focus, we conducted a genetic structure study using microsatellites to assess genotypes circulating in both tsetse flies and domestic animals.

Method: For this study, pyramidal traps were set up and 2695 tsetse flies were collected and 1535 (57\%) living flies were dissected and their mid-guts collected. Furthermore, blood samples were collected from 397 domestic animals (pigs, goats, sheep and dogs). DNA was extracted from midguts and blood samples, and specific primers were used to identify trypanosomes of the subgenus Trypanozoon. All positive samples were genetically characterized with seven microsatellite markers.

Results: Seventy five (4.7\%) midguts of tsetse flies and 140 (35.2\%) domestic animals were found infected by trypanosomes of the subgenus Trypanozoon. The genetic characterization of 215 Trypanozoon positive samples ( 75 from tsetse and 140 from animals) revealed a genetic diversity between Trypanosoma brucei circulating in tsetse and domestic animals. Of these positive samples, 87 (40.5\%) single infections were used here to investigate the population genetics of Trypanosoma brucei circulating in tsetse and domestic animals. The dendrogram illustrating the genetic similarities between Trypanosoma brucei genotypes was subdivided into four clusters. The samples from tsetse belonged to the same cluster whereas the samples from domestic animals and espcially pigs were distributed in the four clusters.

Conclusion: Pigs appeared as the animal species harboring the highest number of different Trypanosoma brucei strains. They may play an important role in the propagation of different genotypes. The $F_{\text {ST }}$ values revealed a sub structuration of Trypanosoma brucei according to hosts and sometimes villages. The data obtained from this study may have considerable importance for the understanding of the transmission and the spread of specific genotypes of Trypanosoma brucei.
\end{abstract}

Keywords: Trypanosoma brucei, Tsetse flies, Domestic animals, Microsatellite markers, Population genetics

\footnotetext{
* Correspondence: gsimoca@yahoo.fr

${ }^{1}$ Department of Biochemistry, Faculty of science, University of Dschang, PO

Box 67, Dschang, Cameroon

Full list of author information is available at the end of the article
} 


\section{Background}

Human African Trypanosomiasis (HAT) also known as sleeping sickness is an important public health disease in sub-Saharan Africa. About 60 million people are at risk, with 10000 new cases reported yearly, with new estimated cumulative infection cases of about 50000 to 70000 [1]. The causative agent of HAT is a protozoan parasite belonging to Trypanosoma brucei (T. brucei) species. This parasite is transmitted by tsetse flies of the genus Glossina. T. brucei infects human as well as a variety of domestic and wild animals in sub-Saharan Africa. Three subspecies of $T$. brucei are currently recognized [2]: Trypanosoma brucei brucei (T. b. brucei), which is defined as infecting animals but not humans and is present throughout the tsetse distribution area in Africa; Trypanosoma brucei gambiense (T. b. gambiense), which is infective for humans in West and Central Africa and whose infection results in chronic human sleeping sickness; and Trypanosoma brucei rhodesiense (T. b. rhodesiense), which is defined as human infective and localized in East and South Africa and causes the acute form of sleeping sickness.

During the last few decades, there has been considerable effort to genetically characterize $T$. brucei isolated from vertebrates and tsetse flies in order to understand the impact of the genetic diversity of trypanosomes on the epidemiology of HAT. Most of these studies were performed on parasite strains isolated from tsetse and mammals. From such strains, unique isolates were obtained and their complete genotypes were easily obtained. However, during the isolation process, a sub sampling of existing genetic diversity occurs inside the host [3], resulting in a possible information loss. The need to undertake investigations on field samples without isolation was a challenge until recently when microsatellite markers were developed. The microsatellite DNA sequences are simple sequence repeats (SSRs), which are hyper-variable, ubiquitous and co-dominant [4]. They occur randomly and abundantly in eukaryotic genomes [5] and are widely used in genetics and phylogenetic studies $[4,6]$. For the subgenus Trypanozoon, a panel of several microsatellite markers has been identified during the last two decades. These markers have been widely used for the molecular characterization of trypanosomes, the assessment of the population structure and reproductive mode [3,7-9], and the construction of the genetic map of trypanosomes [10,11]. The higher sensitivity and specificity of these markers enabled us to genetically characterize $T$. brucei directly from biological samples such as tsetse mid-guts, blood, cerebrospinal fluid and lymph [3,12-14]. Using these biological fluids, the microsatellite markers provided data that permitted us to better understand some biological, genetic and epidemiological aspects of HAT. For instance, the microsatellite markers revealed higher levels of mixed infections of different T. brucei genotypes in tsetse mid-guts [12]. They also revealed a genetic diversity between strains of T. b. gambiense circulating in different fluids of the same HAT patient [13] and multiple infections of T. b. gambiense in the blood and cerebrospinal fluid of HAT patients of Angola [14]. Despite the considerable number of data generated from the molecular characterization of T. brucei, few investigations have been undertaken to characterize the trypanosomes circulating in tsetse flies and mammals of the same locality. It is obvious that such investigations are important to better understand the circulation, the transmission and the spread of different genotypes of trypanosomes.

During the last decade, investigations on the genetic characterization of $T$. brucei circulating in tsetse flies and domestic animals of the Fontem sleeping sickness focus of Cameroon revealed a wide range of $T$. brucei genotypes as well as a high number of mixed genotypes of $T$. brucei in tsetse flies and domestic animals [12,15]. Despite the genetic diversity revealed by these studies, our knowledge of the transmission of different genotypes between trypanosome hosts remains incomplete. Detailed knowledge on the trypanosome genotypes that circulate between tsetse and mammals appears crucial for a better understanding of the population genetics, transmission, and distribution of T. brucei genotypes in different hosts and also for control operations.

For this study, single infections of T. brucei were extracted from data published by Simo et al. [12,15] and then analyzed in order to improve our knowledge on the population genetics of this parasite, and to understand the transmission as well as the circulation of different $T$. brucei genotypes between tsetse flies and domestic animals of the Fontem sleeping sickness focus of Cameroon.

\section{Methods \\ Study area}

The Fontem sleeping sickness focus $\left(05^{\circ} 40^{\prime} 12^{\prime \prime} \mathrm{N}\right.$, $09^{\circ} 55^{\prime} 33^{\prime \prime} \mathrm{E}$ ) is located in the Lebialem, Manyu and Koupe-Manengouba Divisions of the southwest region of Cameroon. This focus is characterized by a tropical humid climate with varied topography of hills and valleys through which several high speed rivers flow. This focus is subdivided into three sub foci: the north, the center and the south sub foci [16]. The main population activities are subsistence agriculture, palm oil extraction, animal husbandry and small scale poultry farming. The dense population of humans, domestic animals and tsetse flies are found scattered in the pre-forest/forest vegetation of the valleys and hills [17]. Glossina palpalis palpalis is the main vector of HAT in this focus [18]. Several domestic animal species including dog, pigs, sheep, and goat are found in this focus. 


\section{Entomological surveys}

For this study, two entomological surveys were carried out in 5 villages (Besali, Bechati, Folepi, Agong and Menji) of the Fontem HAT focus [12]. The first survey was performed in November 2006 and the second in April 2007. In each village, pyramidal traps [19] were deployed in various tsetse fly favorable biotopes. The trapping procedure and the dissection of flies were performed as described in Simo et al. [12].

\section{Blood collection and parasitological analyses}

The domestic animals were sampled during two field surveys in the Fontem HAT focus: the first survey was performed in July 2006 and the second in June 2007. The sampling was carried out in eight villages: Besali, Bechati, Folepi and Agong in the northern sub focus and Nsoko, Fossung, Menji and Azi in the central sub focus [15]. In each village, all domestic animals that had spent at least 3 months in the study zone were selected. All pigs and dogs sampled in this study were of local breed, originating from a mixture of different breeds. The sheep and goats were dwarf breeds. Some pigs were kept in pigsties whereas the others, as well as the other animals were allowed to move freely around the villages.

Sensitization, the blood collection procedures and the parasitological tests have been already described in Simo et al. [15].

\section{DNA extraction}

In the laboratory, the alcohol used to preserve tsetse mid-guts contained in micro-tubes was evaporated for 60 minutes in an oven set at $80^{\circ} \mathrm{C}$. Subsequently, $300 \mu \mathrm{l}$ of Chelex 5\% [20] was added to each tube and the mixture vortexed for 10 minutes. Thereafter, the tubes were incubated first at $56^{\circ} \mathrm{C}$ for one hour, and then at $100^{\circ} \mathrm{C}$ for 30 minutes. The tubes were centrifuged at $14000 \mathrm{rpm}$ for 10 minutes and the supernatant (DNA extract) was collected and stored at $-20^{\circ} \mathrm{C}$ until used.

For the blood samples collected in domestic animals, DNA was extracted from $1 \mathrm{ml}$ of blood using the kit "DNeasy Tissue kit" (Qiagen) as described by Simo et al. [15]. The DNA extract was used directly for PCR or stored at $-20^{\circ} \mathrm{C}$.

Identification of trypanosomes belonging to Trypanozoon This identification was performed using the Trypanozoon specific primers TBR1/2 [21], as described by Simo et al. [16]. The amplification reactions were performed in a final volume of $25 \mu \mathrm{l}$ containing $5 \mu \mathrm{l}$ of DNA extracts. At the end of each amplification reaction, $10 \mu \mathrm{l}$ of each amplified product was resolved on $2 \%$ agarose gel. All TBR1/2 positive DNA samples were used further for the subsequent genetic characterization by microsatellite markers.

\section{Genetic characterization of TBR $1 / 2$ positive DNA samples using microsatellite DNA markers}

All TBR1/2 positive DNA samples (originating from tsetse flies and domestic animals) were genetically characterized using seven microsatellite markers [3,7]. For the samples originating from domestic animals, the amplification reactions were performed in a final volume of $15 \mu \mathrm{l}$ containing $3 \mu \mathrm{l}$ of DNA extract whereas for samples coming from tsetse mid-guts, the amplification reactions were performed in a final volume of $25 \mu \mathrm{l}$ containing $5 \mu \mathrm{l}$ of DNA extract. Whatever the origin of DNA samples, the amplifications were performed in two rounds for the M6C8 and MT3033 markers; the first round was done as described by Biteau et al. [7]. During the second round, $1 \mu \mathrm{l}$ of the amplified product of the first PCR was used as DNA template. For the other markers (Micbg1, Micbg5, Micbg6, Misatg4 and Misatg9), the amplification conditions were identical to those described by Koffi et al. [3]. The amplified products were checked by electrophoresis on $2 \%$ agarose gel and thereafter, the allelic profiles were obtained through an electrophoresis on $10 \%$ non-denaturing acrylamide gels as described in Simo et al. [12,15].

A sample showing more than two alleles was considered here as having multiple infections because $T$. brucei species is a diploid organism and must have one allele or one DNA fragment (homozygote) or two alleles (heterozygote) after the resolution of the amplified products of each microsatellite locus.

\section{Data analysis}

For this analysis, the samples showing more than two alleles or having multiple genotypes were excluded. We considered here as samples with multiple genotypes all samples for which at least three alleles (three DNA fragments) were identified for at least one microsatellite marker. The genetics structure within T. brucei populations circulating in tsetse flies and domestic animals was assessed through Wright's F-statistics [22]. $F_{\mathrm{ST}}$ is a measure of deviation from random distribution of individuals between subsamples (and thus, populations' differentiation). For the differentiation analysis of the populations, the total sample (87 individuals) was first subdivided into five subsamples; each subsample corresponding to one of the five hosts of trypanosomes (tsetse, dog, sheep, goat and pig). Thereafter, a second subdivision into eight subsamples was performed; each subsample here corresponding to villages where tsetse flies and animals were sampled. Wright's F-statistics were estimated using Weir and Cockerham's unbiased estimators [23] in Fstat 2.9.4 software ([24] updated from Goudet [25]). $F_{\mathrm{ST}}$ is a convenient measure of differentiation among the different subsamples of a data set. Its estimator is expected around 0 under the null hypothesis of random distribution of genotypes across 
sub-samples. On the contrary, $F_{\mathrm{ST}}$ estimator displays positive values, up to 1 , in case of genetic differences. For $F_{\mathrm{ST}}$, the estimator was $\theta$ and its significance tested through 10000 permutations of individuals between subsamples. To get an overall idea of the distribution of individuals across hosts and villages, an unrooted NJTREE was computed by the MEGA 3.1 software [26], using the Cavalli-Sforza and Edwards [27] chord distances matrix, which were computed in the GENETIX version 4.05 software package.

The genetic diversity within $T$. brucei populations that circulate in tsetse flies and domestic animals was measured with Nei's unbiased estimator Hs for each locus, over all loci and for multilocus genotypes (MLGs) [28]. All tests and population genetic measures were undertaken with Fstat 2.9.4 ([24], updated from Goudet [25]). All differentiation tests were repeated with the multilocus genotypes (MLGs) where each MLG is coded as an allele of a unique locus as described by Nébavi et al. [29].

\section{Ethical approval}

This study was carried out in strict accordance with the recommendations in the Guide for the Care and Use of Animals of the Department of animal biology and physiology of the Faculty of Science of the University of Yaoundé 1 of Cameroon.

\section{Results}

This study involves 2695 tsetse flies caught in five villages and 397 domestic animals sampled in eight villages.

\section{Identification of Trypanozoon in tsetse mid-guts and their genetic characterization}

During two entomological surveys, 2695 Glossina palpalis palpalis were caught and a total of 1596 (59.2\%) flies were dissected and 352 teneral flies identified. Details concerning entomological results can be found in Simo et al. [12]. The specific PCR targeting a multi-copy 177 bp repeat sequence of trypanosomes of the subgenus Trypanozoon revealed 75 (4.7\%) mid-guts infected by trypanosomes of this subgenus. The genetic characterization was performed on these 75 Trypanozoon positive samples with seven microsatellite markers. Only 5 (Micbg1, M6C8, MT3033, Misatg4 and Misatg9) of the 7 microsatellite markers showed amplifications; no amplification for all the 75 Trypanozoon positive samples was obtained for the markers Micbg5 and Micbg6 [12]. The characteristics of each sample and the allele size for each marker are reported in Simo et al. [12]. Of the 75 samples analyzed, about $41.3 \%$ were multiple infections and therefore, were not considered in the present study since they cannot be included in the population genetic studies. The current study targets 44 samples (single infections) for which no more than two alleles were observed (Table 1). Details concerning the characteristics of each of the 44 samples and the allelic profiles for each marker are reported in Table 1.

\section{Identification of Trypanozoon in domestic animals and their genetic characterization}

The 397 domestic animals sampled in this study include 225 pigs, 87 goats, 65 sheep and 20 dogs. The origins of these animals as well as their parasitological status are reported in Simo et al. [15]. The specific PCR targeting a multi-copy 177 bp repeat sequence of trypanosomes of the subgenus Trypanozoon revealed 140 (35.3\%) animals infected by these trypanosomes. Although the primers used here are able to identify all trypanosomes of the subgenus Trypanozoon, only T. $b$. gambiense and mainly T. $b$. brucei have been identified in animals and tsetse flies of this region $[16,18]$.

The seven microsatellite markers used for the mid-gut infections were also used for the genetic characterization of the 140 Trypanozoon positive samples from animals. Most of the 140 samples were amplified by the seven markers. However, to evaluate the circulation of T. brucei genotypes between tsetse and domestic animals, only the 5 markers for which amplified products were obtained for the 75 Trypanozoon positive samples originating from tsetse flies were considered here. Of the 140 Trypanozoon positive samples originating from domestic animals, 97 were considered as having multiple trypanosome genotypes. Therefore, only 43 samples were considered for this study. The details concerning the characteristics of each of these samples and the size of alleles at each microsatellite locus are reported in Table 2.

A total of 215 Trypanozoon positive samples were identified in tsetse flies and domestic animals. Of these samples, 87 were single infections. For the five markers considered here, about 71 alleles were identified in both multiple and single infections. Twenty (28\%) of these alleles could not be considered for this analysis because they were always found in multiple infections. Most of these alleles were found in a very few number of samples and could thus be considered as belonging to minor genotypes. For instance, alleles such as 158, 145 and 124 of MT3033 were identified only in one sample of multiple infections. It is also the case for allele 75 of M6C8, 90 and 87 of Micbg1.

The number of alleles revealed for each locus was higher in domestic animals than tsetse flies (Table 3). Most alleles found in tsetse were identified in animals. Twelve alleles were identified in tsetse flies and all species of domestic animals. For instance, the alleles such as 142, 156 and 162 of MT3033, 86 of Misatg9, 85 and 91 of M6C8 were identified in tsetse flies and all domestic animal species (Additional file 1: Figure S1). However, some alleles such as 83 of M6C8, 166 of Micbg1, 170 
Table 1 Characteristics of mid-gut single infections of $T$. brucei and size of alleles at each microsatellite locus

\begin{tabular}{|c|c|c|c|c|c|c|c|c|}
\hline \multirow{2}{*}{ Sample code } & \multirow{2}{*}{ Villages } & \multirow{2}{*}{ Tsetse species } & \multicolumn{6}{|c|}{ Size of alleles at each microsatellite locus } \\
\hline & & & Sex & M6C8 & MT3033 & Micbg1 & Misatg4 & MisatG9 \\
\hline $\mathrm{T} 2261$ & Bechati & G. pal & $\mathrm{F}$ & $115 / 105$ & $162 / 142$ & $150 / 166$ & $136 / 128$ & $000 / 000$ \\
\hline T2259 & Bechati & G. pal & $\mathrm{F}$ & $115 / 105$ & $162 / 142$ & $150 / 136$ & $136 / 128$ & 000/000 \\
\hline $\mathrm{T} 2310$ & Bechati & G. pal & M & $115 / 105$ & $162 / 142$ & $150 / 136$ & $136 / 128$ & $170 / 170$ \\
\hline T2262 & Bechati & G. pal & M & $115 / 105$ & $162 / 142$ & $150 / 136$ & $136 / 128$ & $170 / 170$ \\
\hline T105 & Bechati & G. pal & F & $115 / 105$ & $162 / 142$ & $150 / 136$ & $136 / 128$ & $172 / 170$ \\
\hline T177 & Bechati & G. pal & M & $115 / 105$ & $162 / 142$ & $150 / 136$ & $136 / 128$ & 172/170 \\
\hline $\mathrm{T} 2180$ & Bechati & G. pal & F & $115 / 105$ & $162 / 142$ & $150 / 136$ & $136 / 128$ & 170/086 \\
\hline T2362 & Bechati & G. pal & F & $115 / 105$ & $162 / 142$ & $150 / 136$ & $136 / 128$ & $170 / 170$ \\
\hline $\mathrm{T} 2271$ & Bechati & G. pal & F & $115 / 105$ & $162 / 142$ & $150 / 136$ & $136 / 128$ & $170 / 170$ \\
\hline T2247 & Bechati & G. pal & M & $115 / 105$ & $162 / 142$ & $150 / 136$ & $136 / 128$ & 170/170 \\
\hline T2205 & Bechati & G. pal & F & $115 / 105$ & $162 / 142$ & $150 / 136$ & $136 / 128$ & 170/170 \\
\hline T35 & Besali & G. pal & M & 091/085 & $166 / 156$ & $174 / 162$ & $126 / 116$ & 172/170 \\
\hline T39 & Besali & G. pal & F & 091/085 & $166 / 156$ & $174 / 162$ & $126 / 116$ & 172/170 \\
\hline T239 & Folepi & G. pal & M & $115 / 105$ & $162 / 142$ & $150 / 136$ & $136 / 128$ & 170/086 \\
\hline T218 & Folepi & G. pal & M & $115 / 105$ & $162 / 142$ & $150 / 136$ & $136 / 128$ & 000/000 \\
\hline Т1823 & Folepi & G. pal & M & $115 / 105$ & $162 / 142$ & $150 / 136$ & $136 / 128$ & $000 / 000$ \\
\hline Т1075 & Folepi & G. pal & M & $115 / 105$ & $162 / 142$ & $150 / 136$ & $136 / 128$ & $000 / 000$ \\
\hline Т1970 & Folepi & G. pal & F & $115 / 105$ & $162 / 142$ & $150 / 136$ & $136 / 128$ & $170 / 170$ \\
\hline T306 & Folepi & G. pal & $F$ & $115 / 105$ & $162 / 142$ & $150 / 136$ & $136 / 128$ & 170/170 \\
\hline T316 & Folepi & G. pal & F & 095/083 & $162 / 142$ & $150 / 136$ & $136 / 128$ & 172/170 \\
\hline T345 & Folepi & G. pal & M & $115 / 105$ & $162 / 142$ & $150 / 136$ & $136 / 128$ & 170/170 \\
\hline T441 & Folepi & G. pal & $F$ & $115 / 105$ & $162 / 142$ & $150 / 136$ & $136 / 128$ & 172/170 \\
\hline T443 & Folepi & G. pal & M & $115 / 105$ & $162 / 142$ & $150 / 136$ & $136 / 128$ & 170/170 \\
\hline T357 & Folepi & G. pal & M & 091/085 & $166 / 156$ & $174 / 162$ & $126 / 116$ & $000 / 000$ \\
\hline Т1855 & Folepi & G. pal & F & $115 / 105$ & $162 / 142$ & $150 / 136$ & $136 / 128$ & $000 / 000$ \\
\hline T1344 & Folepi & G. pal & M & $115 / 105$ & $162 / 142$ & $150 / 136$ & $136 / 128$ & $000 / 000$ \\
\hline T1005 & Folepi & G. pal & F & $115 / 105$ & $162 / 142$ & $150 / 136$ & $136 / 128$ & $000 / 000$ \\
\hline T1539 & Folepi & G. pal & F & $115 / 105$ & $162 / 142$ & $150 / 136$ & $136 / 128$ & 172/170 \\
\hline $\mathrm{T} 1710$ & Folepi & G. pal & M & $115 / 105$ & $162 / 142$ & $150 / 136$ & $136 / 128$ & 170/170 \\
\hline T1688 & Folepi & G. pal & $\mathrm{F}$ & $115 / 105$ & $162 / 142$ & 150/136 & $136 / 128$ & 000/000 \\
\hline T1947 & Folepi & G. pal & F & $115 / 105$ & $162 / 142$ & $150 / 136$ & $136 / 128$ & $000 / 000$ \\
\hline T2066 & Folepi & G. pal & F & $115 / 105$ & $162 / 142$ & $150 / 136$ & $136 / 128$ & $000 / 000$ \\
\hline T1954 & Folepi & G. pal & F & $115 / 105$ & $162 / 142$ & $150 / 136$ & $136 / 128$ & 170/086 \\
\hline T1506 & Folepi & G. pal & F & $115 / 105$ & $162 / 142$ & $150 / 136$ & $136 / 128$ & $172 / 170$ \\
\hline T1743 & Folepi & G. pal & M & $115 / 105$ & $162 / 142$ & $150 / 136$ & $136 / 128$ & $000 / 000$ \\
\hline T1347 & Folepi & G. pal & M & $115 / 105$ & $162 / 142$ & $150 / 136$ & $136 / 128$ & 172/170 \\
\hline Т1653 & Folepi & G. pal & F & $115 / 105$ & $162 / 142$ & $150 / 136$ & $136 / 128$ & 172/170 \\
\hline T1646 & Folepi & G. pal & F & 091/085 & $162 / 142$ & 150/136 & $136 / 128$ & 136/134 \\
\hline T2029 & Folepi & G. pal & F & $115 / 105$ & $162 / 142$ & $150 / 136$ & $136 / 128$ & 172/170 \\
\hline T2093 & Folepi & G. pal & F & $115 / 105$ & $162 / 142$ & $150 / 136$ & $136 / 128$ & 170/170 \\
\hline T2445 & Menji & G. pal & $\mathrm{F}$ & 091/085 & $166 / 156$ & $174 / 162$ & $126 / 116$ & 170/086 \\
\hline
\end{tabular}


Table 1 Characteristics of mid-gut single infections of $T$. brucei and size of alleles at each microsatellite locus (Continued)

\begin{tabular}{|c|c|c|c|c|c|c|c|c|}
\hline T2482 & Menji & G. pal & $\mathrm{F}$ & $115 / 105$ & $162 / 142$ & $150 / 136$ & $136 / 128$ & $000 / 000$ \\
\hline T2481 & Menji & G. pal & $\mathrm{F}$ & $115 / 105$ & $162 / 142$ & $150 / 136$ & $136 / 128$ & $170 / 170$ \\
\hline T2427 & Menji & G. pal & $\mathrm{F}$ & $115 / 105$ & $162 / 142$ & $150 / 136$ & $136 / 128$ & $170 / 170$ \\
\hline
\end{tabular}

G. pal Glossina palpalis palpalis; $F$ female; $M$ male. Results of microsatellite markers are given as: AAA/BBB where AAA is the size in base pair of the largest allele and $\mathrm{BBB}$, the size of the smallest allele. 000/000: no amplification.

and 172 of Misatg9 were identified only in tsetse flies. Furthermore, some alleles found in domestic animals were not identified in tsetse flies (Additional file 1: Figure S1).

Eighty-seven single infections of T. brucei including 44 originating from tsetse flies and 43 from domestic animals were selected for the genetic analyses (Tables 1 and 2). Genotypes of 87 T. brucei positive samples were scored at 5 microsatellite loci. These markers showed some degree of genetic diversity within $T$. brucei populations circulating in tsetse flies and domestic animals of the Fontem HAT focus. The value of the genetic diversity ranges from 0.369 (MISATG locus) to 0.888 (MT3033 locus), with an overall value of 0.695 (Table 4). All the microsatellite markers were highly polymorphic with a substantial number of different multilocus genotypes (MLGs).

\section{Neighbour-joining analysis}

To examine the level of the genetic diversity in the population of $T$. brucei, multilocus genotypes were derived for each sample from the genotyping performed with the five microsatellite markers considered for the present study. Thereafter, a dendrogram of similarity was constructed from the data obtained. A total of 40 distinct multilocus genotypes were identified from the 87 samples considered here. The dendrogram generated indicates that the $T$. brucei populations form a large group with a substantial number of different genotypes (Figure 1). This dendrogram can be subdivided into four clusters (Figure 1): the first cluster contains all samples of T. brucei originating from tsetse flies and three additional samples coming from pig (P236 and P606) and goat (G590). The second cluster contains 15 samples of T. brucei originating from pig and two additional samples coming from goat (G281) and sheep (S595). The third cluster contains samples originating mostly from sheep and goats and the last cluster contains samples coming from different species of domestic animals. Considering the distribution of $T$. brucei genotypes in different clusters, it appears that the trypanosomes' hosts can be considered as separate entities for investigations aiming to better understand the genetic diversity within $T$. brucei populations. In this light, subsequent analyses were undertaken with subpopulations defined as all T. brucei positive samples originating from the same host or coming from the same village.

\section{Differentiation of $T$. brucei populations}

Considering the samples originating from the same host as one subpopulation, the pairwise $F_{\mathrm{ST}}$ values between different subpopulations (Table 5) indicate a genetic differentiation between the trypanosomes' hosts (values ranging from 0.0317 to 0.3163 ). Except for the comparisons between trypanosomes extracted from goat and pig, as well as, goat and sheep, which show a lower genetic differentiation $\left(F_{\mathrm{ST}}=0.0797\right.$ and 0.0317 , respectively), the other comparisons show greater genetic differentiation (Table 5). The genetic differentiation between goat and sheep was not significant $(P=0.1318)$ whereas the values obtained for the other comparisons were highly significant $(\mathrm{P}<0.001)$. Moreover, the pairwise $F_{\mathrm{ST}}$ values indicate some genetic differentiations between villages (values ranging from 0.0124 to 0.3080 ). Some villages show very little genetic differentiation while others reveal greater genetic differentiation with significant $p$ values (Table 6). The results of this study show that T. brucei populations of the Fontem HAT focus display diverse genotypes. However, few closely related genotypes circulate most often in tsetse flies.

\section{Discussion}

This study on the population genetics of $T$. brucei revealed a high genetic diversity between $T$. brucei circulating in tsetse flies and domestic animals of the Fontem HAT focus of Cameroon. For this study, multiple infections were excluded because they could not show the genetic information for each individual trypanosome. Such information is needed for population genetic studies like for instance the evaluation of the F statistics. Therefore, some alleles involved in these infections (probably minor genotypes) were not taken into account. Whatever the microsatellite marker, the majority of alleles identified in tsetse flies and domestic animals were selected for this study and consequently, the genotypes analyzed here are probably the major genotypes found most often in the Fontem focus. However, it is important to point out that the exclusion of some alleles and the fact that wild animals (host of trypanosomes and source of blood meals for tsetse flies) were not included in this study have probably led to an underestimation of the real genetic diversity. The identification of T. brucei in tsetse flies and domestic animals of the Fontem HAT confirms results of previous authors $[16,18]$ who identified $T . b$. brucei and T. b. gambiense in 
Table 2 Characteristics of domestic animals' single infections of $T$. brucei and size of alleles at each microsatellite locus

\begin{tabular}{|c|c|c|c|c|c|c|c|}
\hline \multirow{2}{*}{ Sample code } & \multirow{2}{*}{ Animal species } & \multirow{2}{*}{ Village } & \multicolumn{5}{|c|}{ Microsatellite markers } \\
\hline & & & M6C8 & MT3033 & MICBG1 & MISATG4 & MISATG9 \\
\hline P302 & Pig & Agong & $175 / 175$ & $162 / 142$ & 190/190 & 190/159 & $234 / 086$ \\
\hline P303 & Pig & Agong & $000 / 000$ & $310 / 310$ & $190 / 190$ & $221 / 221$ & 086/086 \\
\hline P304 & Pig & Agong & $175 / 175$ & $162 / 142$ & $190 / 190$ & 190/116 & $234 / 086$ \\
\hline P306 & Pig & Agong & $175 / 175$ & $310 / 310$ & 190/190 & $221 / 149$ & 086/086 \\
\hline P309 & Pig & Agong & $175 / 175$ & $162 / 142$ & $190 / 136$ & $221 / 149$ & 086/086 \\
\hline P310 & Pig & Agong & $000 / 000$ & $162 / 142$ & 190/190 & $221 / 149$ & $234 / 086$ \\
\hline S595 & Sheep & Agong & $115 / 150$ & $162 / 142$ & 190/190 & $123 / 116$ & 086/086 \\
\hline S596 & Sheep & Agong & $115 / 150$ & $162 / 142$ & 190/190 & $221 / 149$ & $242 / 242$ \\
\hline$S 602$ & Sheep & Agong & 091/085 & $208 / 208$ & $136 / 136$ & $000 / 000$ & $242 / 242$ \\
\hline P236 & Pig & Bechati & $115 / 105$ & $162 / 142$ & 200/190 & $190 / 136$ & $000 / 000$ \\
\hline P240 & Pig & Bechati & $175 / 175$ & $162 / 142$ & 190/190 & $190 / 128$ & $234 / 234$ \\
\hline P245 & Pig & Bechati & $175 / 175$ & $310 / 310$ & 190/190 & 159/136 & 086/086 \\
\hline G246 & Goat & Bechati & 091/085 & $000 / 000$ & $150 / 150$ & $221 / 221$ & $234 / 086$ \\
\hline P247 & Pig & Bechati & $175 / 175$ & $310 / 310$ & 200/190 & $128 / 128$ & 086/086 \\
\hline S255 & Sheep & Bechati & $275 / 275$ & $310 / 200$ & $162 / 108$ & $116 / 116$ & $234 / 234$ \\
\hline G281 & Goat & Bechati & 091/085 & $162 / 142$ & 190/108 & $165 / 128$ & 086/086 \\
\hline G546 & Goat & Bechati & 091/085 & $000 / 000$ & 190/108 & $165 / 128$ & $242 / 242$ \\
\hline G548 & Goat & Bechati & 091/085 & $310 / 200$ & $224 / 108$ & $165 / 128$ & $242 / 242$ \\
\hline G550 & Goat & Bechati & 091/085 & $162 / 142$ & 190/108 & $000 / 000$ & 086/086 \\
\hline G590 & Goat & Bechati & $301 / 301$ & 000/000 & $162 / 115$ & $136 / 128$ & 136/086 \\
\hline G593 & Goat & Bechati & 091/085 & $310 / 200$ & $174 / 150$ & $000 / 000$ & $242 / 242$ \\
\hline S219 & Sheep & Besali & $275 / 275$ & $310 / 200$ & $250 / 190$ & $221 / 110$ & $234 / 234$ \\
\hline S221 & Sheep & Besali & $275 / 275$ & $200 / 200$ & 234/190 & $165 / 165$ & $234 / 234$ \\
\hline P229 & Pig & Besali & $275 / 175$ & $200 / 200$ & 220/190 & $128 / 116$ & $234 / 234$ \\
\hline P301 & Pig & Folepi & $175 / 175$ & $162 / 142$ & 190/190 & $126 / 126$ & $234 / 086$ \\
\hline D572 & Dog & Folepi & $000 / 000$ & $000 / 000$ & $000 / 000$ & $000 / 000$ & $242 / 176$ \\
\hline S587 & Sheep & Folepi & $115 / 070$ & $156 / 142$ & $190 / 190$ & $000 / 000$ & $242 / 242$ \\
\hline P676 & Pig & Fossung & $179 / 175$ & $310 / 142$ & $126 / 126$ & $000 / 000$ & 086/086 \\
\hline P334 & Pig & Menji & $175 / 175$ & $166 / 156$ & $174 / 162$ & $221 / 116$ & 086/086 \\
\hline P335 & Pig & Menji & $175 / 175$ & $166 / 156$ & 200/190 & $116 / 116$ & $234 / 086$ \\
\hline P605 & Pig & Menji & $179 / 175$ & $310 / 142$ & $190 / 190$ & $221 / 221$ & $242 / 242$ \\
\hline P606 & Pig & Menji & 091/085 & $310 / 142$ & $136 / 136$ & $149 / 136$ & $242 / 234$ \\
\hline P624 & Pig & Menji & 091/085 & $310 / 142$ & $136 / 115$ & $123 / 128$ & 086/086 \\
\hline P631 & Pig & Menji & 091/085 & $310 / 142$ & $174 / 162$ & $149 / 136$ & 086/086 \\
\hline P641 & Pig & Menji & 091/085 & $310 / 142$ & $250 / 190$ & $221 / 221$ & $128 / 086$ \\
\hline P643 & Pig & Menji & 091/085 & $310 / 142$ & $174 / 162$ & $221 / 221$ & 086/086 \\
\hline P645 & Pig & Menji & 091/085 & $310 / 142$ & $174 / 162$ & $123 / 128$ & $128 / 086$ \\
\hline P648 & Pig & Menji & $179 / 175$ & $310 / 142$ & $220 / 115$ & $221 / 221$ & $128 / 086$ \\
\hline G353 & Goat & Nsoko & $275 / 275$ & 187/156 & 190/108 & $221 / 149$ & $234 / 086$ \\
\hline G355 & Goat & Nsoko & $275 / 275$ & $166 / 156$ & 190/108 & $221 / 149$ & $234 / 086$ \\
\hline P658 & Pig & Nsoko & $179 / 175$ & $310 / 142$ & $174 / 136$ & $149 / 116$ & $128 / 086$ \\
\hline
\end{tabular}


Table 2 Characteristics of domestic animals' single infections of $T$. brucei and size of alleles at each microsatellite locus (Continued)

\begin{tabular}{lllllll}
\hline G665 & Goat & Nsoko & $091 / 085$ & $310 / 142$ & $190 / 115$ & $000 / 000$ \\
P672 & Pig & Nsoko & $179 / 175$ & $162 / 142$ & $136 / 115$ & $123 / 116$ \\
S390 & Sheep & Azi & $091 / 085$ & $200 / 187$ & $190 / 108$ & $116 / 116$
\end{tabular}

Results of microsatellite markers are given as: AAA/BBB where AAA is the size in base pair of the largest allele and BBB, the size of the smallest allele. 000/000: no amplification.

tsetse flies and mammals (human and animals) of this region. The previous identification of T. $b$. gambiense at a very low prevalence in patients $(0.05 \%$ in human for instance [16]) and domestic animals suggests that most strains analyzed in this study belong to T. b. brucei.

For the five microsatellite markers considered here, more alleles were identified in animals compared to tsetse flies (Table 3); thus indicating a high level of genetic diversity in T. brucei strains circulating in animals compared to those found in tsetse flies. These results are in line with the observations of MacLeod et al. [30] that the full level of T. brucei diversity is only apparent when tsetse flies are examined. The fact that some genotypes circulating in animals (minor genotypes) could not be found in tsetse mid-guts is difficult to explain because the mid-gut genotypes (immature infections) probably come from infected animals. Nevertheless, some minor genotypes that cannot be observed in the midgut can develop to maturation in the salivary glands, and therefore, can be transmitted to animals. In such context, the identification of some genotypes in animals, but not in tsetse flies could be explained by the fact that several infected tsetse flies with different $T$. brucei genotypes can feed simultaneously or successively on the same animal [15]. During their blood meals, infected flies can transmit different T. brucei genotypes to the vertebrate host. Another explanation may result from the bottlenecks observed during the development of trypanosomes in tsetse flies [31]. These bottlenecks may lead to a considerable number of minor genotypes, which are able to escape detection in tsetse mid guts. Furthermore, some minor mid gut trypanosome populations can be amplified in the salivary glands of

Table 3 Number of alleles for each locus and according to different hosts of trypanosomes

\begin{tabular}{lccccc}
\hline & \multicolumn{5}{c}{ Number of alleles sampled per population } \\
\cline { 2 - 6 } Loci & Tsetse & Goat & Pig & Sheep & Total \\
\hline MICBG1 & 5 & 7 & 9 & 6 & 14 \\
MISATG & 4 & 5 & 9 & 6 & 11 \\
MISATG & 5 & 5 & 4 & 3 & 9 \\
M6C8 & 6 & 4 & 7 & 6 & 12 \\
MT3033 & 4 & 7 & 6 & 7 & 8 \\
Total & 24 & 28 & 35 & 28 & 54 \\
\hline
\end{tabular}

individual tsetse flies [31]. These minor genotypes could be disseminated to vertebrate hosts, resulting probably in the rapid spread of new genotypes in vertebrate hosts [15]. Another possible outcome of the bottleneck is that some rare variants can be amplified in individual flies and disseminated by them to their vertebrate hosts [32]. If such variants have some selective advantages in mammals, such as altered host range or increased resistance to drugs, this might cause them to become the major variants or genotypes circulating locally $[33,34]$. Amongst the animals, pigs appear as the species infected by several strains of trypanosomes because 35 different alleles were identified in pigs and about 18 genotypes belonging to the four clusters were also identified in these animals. In addition, almost all genotypes or closely related genotypes were identified in pigs. Pigs therefore appear as the animal species that are able to play an important role in the propagation and the spread of different T. brucei genotypes. In this context, the characterization of strains circulating in pigs could give a real indication of the genetic diversity of $T$. brucei circulating in this region. The high number of genotypes circulating in pigs could be linked to the feeding preference of tsetse flies since about $55 \%$ of tsetse blood meals collected in this region were from pigs [35].

Out of 71 alleles (multiple and single infections) revealed in this study, only 12 of them were identified in T. brucei found in tsetse flies and domestic animals as well. These results indicate a very low number of identical genotypes that circulate between tsetse and domestic animals in this region. This is strengthened by the three genotypes originating from animals that cluster with those found in tsetse (Figure 1). The presence, in each host (tsetse and animals), of several trypanosome genotypes

Table 4 Value of the genetic diversity at each microsatellite locus

\begin{tabular}{lcc}
\hline Loci & Observed genetic diversity & Expected genetic diversity \\
\hline MICBG1 & 0.740 & 0.710 \\
MISATG & 0.756 & 0.772 \\
MISATG & 0.369 & 0.613 \\
M6C8 & 0.723 & 0.708 \\
MT3033 & 0.888 & 0.762 \\
Overall & 0.695 & 0.713 \\
\hline
\end{tabular}




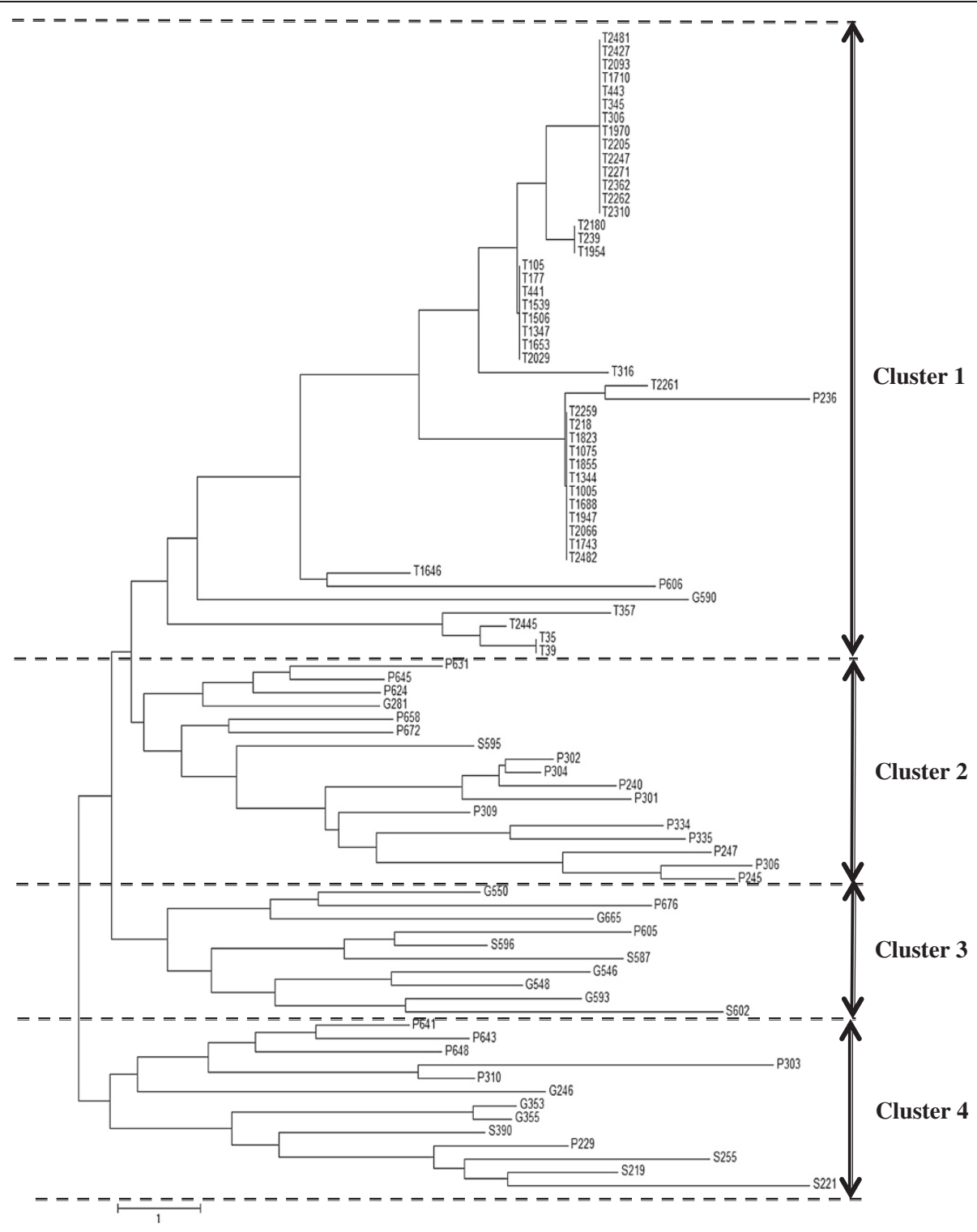

Figure 1 Dendrogram showing the genetic diversity in $T$. brucei circulating in tsetse flies and domestic animals of the sleeping sickness focus of Fontem. P: pig; S: sheep; G: Goat; T: Tsetse flies.

suggests the heterogeneous nature of T. brucei strains circulating in the Fontem HAT focus.

The dendrogram of Figure 1 shows a clustering of T. brucei genotypes into four clusters. The genotypes belonging to the same cluster can be considered as closely related strains or closely related genotypes. The

Table 5 FST values between the hosts of trypanosomes

\begin{tabular}{cccc}
\hline & Goat & Pig & Sheep \\
\hline G. p. pal & $0.2752^{*}$ & $0.3040^{*}$ & $0.3163^{*}$ \\
Goat & & $0.0797^{*}$ & 0.0317 \\
Pig & & & 0.1077 \\
\hline
\end{tabular}

G.p. pal Glossina palpalis palpalis; *: P value significant. identification of several genotypes within the same cluster illustrates the genetic differentiation between closely related strains as reported previously by Simo et al. [9], for T. brucei gambiense. Each cluster can be linked to specific hosts of trypanosomes. For instance, cluster 1 contained strains found mainly in tsetse flies. Within this cluster about 10 different genotypes were identified for 44 samples; thus confirming the low genetic diversity between T. brucei circulating in tsetse flies. These results could be explained in part by the low life expectancy of tsetse flies (cannot live for more than four months). In such a context, tsetse flies cannot accumulate infections in the mid-guts like mammals, which are able to do it in the blood or other corporal fluids. The presence of three 
Table 6 Values of FST between villages

\begin{tabular}{lccccc}
\hline & Bechati & Besali & Folepi & Menji & Nsoko \\
\hline Agong & $0.1485^{*}$ & $0.2143^{*}$ & $0.3140^{*}$ & $0.0858^{*}$ & 0.0555 \\
Bechati & & $0.1530^{*}$ & 0.0403 & 0.0437 & $0.1061^{*}$ \\
Besali & & & $0.3080^{*}$ & 0.1192 & 0.0700 \\
Folepi & & & $0.1678^{*}$ & $0.2742^{*}$ \\
Menji & & & & 0.0124 \\
\hline *. P value significant. & & & &
\end{tabular}

samples from pigs and goat in the cluster 1 confirms the circulation of closely related genotypes between tsetse flies and mammals. In other clusters where the genetic diversity is high with for instance about 12 genotypes identified in cluster 2 for 18 samples, all T. brucei strains came from mammals. This higher genetic diversity observed for the strains of T. brucei that circulate in animals could be explained by the longevity of animals in the tsetse infested region. Indeed, most animals sampled in this region have spent at least three months in the region. During this period, these animals had the possibility to be infected by different strains of $T$. brucei due to cumulative and sequential transmission of different genotypes of T. brucei.

In order to determine if $T$. brucei populations can be sub-structured on the basis of the origin of samples, the $F_{\mathrm{ST}}$ was evaluated by considering a subpopulation as all samples originating from the same host or from the same village. Our results show high value of $F_{\mathrm{ST}}$ between tsetse flies and all animal species. This suggests that most of $T$. brucei strains circulating in tsetse flies are genetically different from strains circulating in domestic animals. Between trypanosomes coming from sheep and goat, the $F_{\mathrm{ST}}$ value was low (0.0317) and statistically not significant, thus suggesting closely related genotypes between these animals. This hypothesis is strengthened by the presence of genotypes from sheep and goat in the same clusters, mainly in cluster 3 and 4 (Figure 1). The $F_{\mathrm{ST}}$ values between pigs and other species is moderate and statistically significant. This suggests also that some strains circulating in pigs were genetically different from those found in other hosts. Our results are in line with previous observations of MacLeod et al. [30] who reported that host selection is an important determinant of the population structure of T. brucei, as particular genotypes of trypanosome are better adapted to survival within different mammalian hosts. It appears that within T. brucei subpopulations, each host must be considered as a separate entity. Despite the fact that the sample size was low for some subpopulations like for instance goat, with only eight samples, the results obtained here suggest a sub-structuration of $T$. brucei according to trypanosomes' hosts.

Looking at the $F_{\mathrm{ST}}$ between villages (Table 6), some values were low and statistically not significant, suggesting little genetic differentiation or no sub-structuration between T. brucei circulating in tsetse flies and animals of some villages of the Fontem focus. However, high and significant $F_{\mathrm{ST}}$ values between villages like Agong and Folepi $\left(F_{\mathrm{ST}}=0.314 ; \mathrm{P}=0.0001\right)$, Folepi and Besali $(\mathrm{FST}=0.308$; $\mathrm{P}=0.0002$ ) indicates that, even in the same focus, considerable genetic differentiation can be observed between T. brucei strains of different villages. Such genetic differentiation may have a real impact on the transmission and the spread of the disease like for instance the most pathogenic strains.

\section{Conclusion}

Microsatellite markers enabled us to better understand the distribution of T. brucei genotypes in tsetse flies and different species of domestic animals of the Fontem HAT focus of southern Cameroon. Amongst different hosts, pig appeared as the animal species, which is infected by several genotypes of T. brucei. The data generated in this study enabled us to cluster $T$. brucei genotypes according to hosts. They also showed a sub structuration of T. brucei according to infected animals and sometimes villages. Our results have considerable importance for the understanding of the transmission and the spread of T. brucei genotypes.

\section{Additional file}

Additional file 1: Figure S1. Frequency of alleles for each microsatellite locus and for each host of T. brucei.

\section{Competing interests}

The authors declare that they have no competing interests.

\section{Authors' contributions}

GS participated in the conception of the study, design of experiments, collected field data and draft the manuscript. NGR collected field data, performed molecular analyses and drafted the manuscript. TTMT helped in the population genetics analysis and the draft the manuscript. GC participated in the conception of the study and helped to draft the manuscript. FN participated in the conception of the study, collected field data and helped to draft the manuscript. TA participated in the conception of the study and helped to draft the manuscript. All authors read and approved the final manuscript.

\section{Acknowledgements}

Gustave Simo was supported by the "Alexander Von Humboldt Stiftung" and the "European Foundation Initiative for NTDs, EFINTD". This study was also supported by AIRES-SUD project $n^{\circ} 7003$, IRD, the University of Yaoundé I and the University of Dschang.

\section{Author details}

${ }^{1}$ Department of Biochemistry, Faculty of science, University of Dschang, PO Box 67, Dschang, Cameroon. ${ }^{2}$ Department of Animal Biology and Physiology, Parasitology and Ecology Laboratory, Faculty of Science, University of Yaounde I, P.O. Box 812, Yaounde, Cameroon. ${ }^{3}$ Institut de Recherche pour le Développement, Unité Mixte de Recherche 177 IRD-CIRAD, Campus International de Baillarguet, TA A17/G, 34398 Montpellier Cedex 5, France. ${ }^{4}$ Faculty of medicine and Biomedical Science, University of Yaoundé 1, Yaounde, Cameroon. 
References

1. Simarro PP, Jannin J, Cattand P: Eliminating human African Trypanosomiasis: Where do we stand and what comes next? PLOS Med 2008, 5:e55.

2. Hoare CA: The Trypanosomes of Mammals: A Zoological Monograph. Oxford: Blackwell Scientific publication; 1972:749. 716.

3. Koffi M, Solano P, Barnabe B, De Meeus T, Bucheton B, Cuny G, Jamonneau V: Genetic characterisation of Trypanosoma brucei s.l. using microsatellite typing: new perspectives for the molecular epidemiology of human African trypanosomosis. Infect Genet Evol 2007, 7:675-684.

4. Schwenkenbecher JM, Frohlich C, Gehre F, Schnur LF, Schonian G: Evolution and conservation of microsatellite markers for Leishmania tropica. Infect Genet Evol 2004, 4:99-105.

5. Hamada H, Petrino MG, Kakunaga T: A novel repeated element with ZDNA- forming potential is widely found in evolutionarily diverse eukaryotic genomes. Proc Natl Acad Sci U S A 1982, 79:6465-6469.

6. Schwenkenbecher JM, Wirth T, Schnur LF, Jaffe CL, Schallig H, Al-Jawabreh A, Hamarsheh O, Kifaya A, Pralong F, Schonian G: Microsatellite analysis reveals genetic structure of Leishmania tropica. Int J Parasitol 2006, 36:237-246.

7. Biteau N, Bringaud F, Gibson W, Truc P, Baltz T: Characterization of Trypanozoon isolates using a repeated coding sequence and microsatellite markers. Mol Biochem Parasitol 2000, 105:185-201.

8. Koffi M, De Meeus T, Bucheton B, Solano P, Camara M, Kaba D, Cuny G, Ayala FJ, Jamonneau V: Population genetics of Trypanosoma brucel gambiense, the agent of sleeping sickness in Western Africa. Proc Nat Acad Sci U S A 2009, 106:209-214.

9. Simo G, Njiokou F, Tume C, Lueong S, De Meeûs T, Cuny G, Asonganyi T: Population genetic structure of Central African Trypanosoma brucei gambiense isolates using microsatellite DNA markers. Infect Genet Evol 2010, 10:68-76.

10. MacLeod A, Tweedie A, McLellan S, Taylor S, Hall N, Berriman M, El-Sayed NM, Hope M, Turner CM, Tait A: The genetic map and comparative analysis with the physical map of Trypanosoma brucei. Nucleic Acids Res 2005 27:6688-6693

11. Cooper A, Tait A, Sweeney L, Tweedie A, Morrison L, Turner CM, MacLeod A: Genetic analysis of the human infective trypanosome Trypanosoma brucei gambiense: chromosomal segregation, crossing over, and the construction of a genetic map. Genome Biol 2008, 9:R103.

12. Simo G, Njitchouang GR, Njiokou F, Cuny G, Asonganyi T: Trypanosoma brucei s.l.: microsatellite markers revealed high level of multiple genotypes in the mid-guts of wild tsetse flies of the Fontem sleeping sickness focus of Cameroon. Exp Parasitol 2011, 128:272-278.

13. Kabore J, Koffi M, Bucheton B, MacLeod A, Duffy C, Ilboudo H, Camara M, De Meeus T, Belem AM, Jamonneau V: First evidence that parasite infecting apparent aparasitemic serological suspects in human African trypanosomiasis are Trypanosoma brucei gambiense and are similar to those found in patients. Infect Genet Evol 2011, 11:1250-1255.

14. Truc $P$, Tiouchichine ML, Cuny G, Vatunga G, Josenando T, Simo G, Herder S: Multiple infections of Trypanosoma brucei gambiense in blood and cerebrospinal fluid of human African trypanosomosis patients from Angola: consequences on clinical course and treatment outcome. Infect Genet Evol 2012, 12:399-402.

15. Simo G, Njitchouang GR, Njiokou F, Cuny G, Asonganyi T: Genetic characterization of Trypanosoma brucei circulating in domestic animals of the Fontem sleeping sickness of Cameroon. Microbes Infect 2012, 14:651-658.

16. Simo G, Asonganyi T, Nkinin SW, Njiokou F, Herder S: High prevalence of Trypanosoma brucei gambiense group 1 in pigs from the Fontem sleeping sickness focus in Cameroon. Vet Parasitol 2006, 139:57-66.

17. Asonganyi T, Suh S, Tetuh MD: Prevalence of domestic animal trypanosomiasis in the Fontem sleeping sickness focus, Cameroon. Rev Elev Med Vet Pays Trop 1990, 43:69-74.

18. Morlais I, Grebaut P, Bodo JM, Djoha S, Cuny G: Characterization of trypanosome infections by polymerase chain reaction (PCR) amplification in wild tsetse flies in Cameroon. Parasitology 1998, 116:547-554.

19. Gouteux JP, Lancien J: The pyramidal trap for collecting and controlling tsetse flies (Diptera: Glossinidae). Comparative trials and description of new collecting techniques. Trop Med Parasitol 1986, 37:61-66.

20. Walsh PS, Metzger DA, Higuchi: Chelex 100 as a medium for simple extraction of DNA for PCR-based typing from forensic material. Biotechniques 1991, 10:506-513.
21. Masiga DK, Smyth AJ, Hayes P, Bromidge TJ, Gibson WC: Sensitive detection of trypanosomes in tsetse flies by DNA amplification. Int J Parasitol 1992, 22:909-918

22. Wright S: The interpretation of population structure by F-statistics with special regard to system of mating. Evolution 1965, 19:395-420.

23. Weir BS, Cockerham CC: Estimating F-statistics for the analysis of population structure. Evolution 1984, 38:1358-1370.

24. Goudet J: Fstat (ver. 2.9.4), A Program to Estimate and Test Population Genetics Parameters; 2003. Available from http://www2.unil.ch/popgen/ softwares/ Updated from Goudet [1995].

25. Goudet J: FSTAT (Version 1.2): a computer program to calculate F-statistics. $J$ Hered 1995, 86:485-486.

26. Kumar S, Tamura K, Nei M: MEGA3: integrated software for molecula evolutionary genetics analysis and sequence alignment. Brief Bioinform 2004, 5:150-163.

27. Cavalli-Sforza LL, Edwards AWF: Phylogenetic analysis: model and estimation procedures. Am J Hum Genet 1967, 19:233-257.

28. Nei M, Chesser RK: Estimation of fixation indices and gene diversities. Ann Hum Genet 1983, 47:253-259.

29. Nebavi F, Ayala FJ, Renaud F, Bertout S, Eholié S, Moussa K, Mallie M, De Meeus T: Clonal population structure and genetic diversity of Candida albicans in AIDS patients from Abidjan (Cote d'Ivoire). Proc Natl Acad Sci U S A 2006, 103:3663-3668

30. MacLeod A, Tweedie A, Welburn SC, Maudlin I, Turner CMR, Tait A Minisatellite marker analysis of Trypanosoma brucei: reconciliation of clonal, panmictic, and epidemic population genetic structures. Proc Nat Acad Sci U S A 2000, 97:13442-13447.

31. Oberle M, Balmer O, Brun R, Roditi I: Bottlenecks and the maintenance of minor genotypes during the life cycle of Trypanosoma brucei. PLOS Pathog 2010, 6:e1001023.

32. Stevens JR, Mathieu Daude F, McNamara JJ, Mizen VH, Nzila A: Mixed populations of Trypanosoma brucei in wild Glossina palpalis palpalis. Trop Med Parasitol 1994, 45:313-318.

33. Wootton JC, Feng X, Ferdig MT, Cooper RA, Mu J, Baruch DI, Magill AJ, Su XZ: Genetic diversity and chloroquine selective sweeps in Plasmodium falciparum. Nature 2002, 418:320-323.

34. De Roode J, Culleton R, Bell A, Read A: Competitive release of drug resistance following drug treatment of mixed Plasmodium chabaudi infections. Malar J 2004, 3:33.

35. Njitchouang GR, Njiokou F, Nana-Djeunga H, Asonganyi T, Fewou-Moundipa $P$ Cuny G, Simo G: A new transmission risk index for human African trypanosomiasis and its application in the identification of sites of high transmission of sleeping sickness in the Fontem focus of southwest Cameroon. Med Vet Entomol 2011, 25:289-296.

doi:10.1186/1756-3305-7-156

Cite this article as: Simo et al:: Population genetics of Trypanosoma brucei circulating in Glossina palpalis palpalis and domestic animals of the Fontem sleeping sickness focus of Cameroon. Parasites \& Vectors 2014 7:156

\section{Submit your next manuscript to BioMed Central and take full advantage of:}

- Convenient online submission

- Thorough peer review

- No space constraints or color figure charges

- Immediate publication on acceptance

- Inclusion in PubMed, CAS, Scopus and Google Scholar

- Research which is freely available for redistribution 